\title{
Nonlinear Bernstein-type operators providing a better error estimation
}

\author{
Oktay Duman
}




\title{
NONLINEAR BERNSTEIN-TYPE OPERATORS PROVIDING A BETTER ERROR ESTIMATION
}

\author{
OKTAY DUMAN
}

Received 18 February, 2014

\begin{abstract}
In this paper, when approximating a continuos non-negative function on the unit interval, we present an alternative way to the classical Bernstein polynomials. Our new operators become nonlinear, however, for some classes of functions, they provide better error estimations than the Bernstein polynomials. Furthermore, we obtain a simultaneous approximation result for these operators.
\end{abstract}

2010 Mathematics Subject Classification: 41A36; 41A28

Keywords: Bernstein polynomials, nonlinear operators, simultaneous approximation

\section{INTRODUCTION}

Polynomials appear in a wide variety of areas of mathematics and science. We often need polynomials rather than more complicated functions as they are simply defined, can be calculated on computer systems and represent a tremendous variety of functions. However, sometimes it may be needed to modify polynomials in order to get a more efficient approximation. This paper concerns with the modification of the classical sequence of Bernstein polynomials (see [1]) which is one of the most widely used tools in approximation theory and numerical analysis. We show that our modified operators become nonlinear with respect to a given function, however, for some classes of functions, they provide better error estimations than the Bernstein polynomials. More precisely, we introduce the following operators:

$$
L_{n, \alpha}(f ; x):=\left\{\sum_{k=0}^{n}\left(\begin{array}{l}
n \\
k
\end{array}\right) x^{k}(1-x)^{n-k} f^{\alpha}\left(\frac{k}{n}\right)\right\}^{1 / \alpha},
$$

where $f$ is a non-negative function on $[0,1], x \in[0,1], n \in \mathbb{N}$ and $\alpha>0$. Observe that

$$
L_{n, \alpha}(f ; x)=\left(B_{n}\left(f^{\alpha} ; x\right)\right)^{1 / \alpha}
$$

where $B_{n}$ denotes the classical Bernstein polynomials. We refer [2] for detailed investigations on the Bernstein polynomials. Observe that $L_{n, 1}(f ; x)=B_{n}(f, x)$. From (1.1), we see that operators $L_{n, \alpha}$ are positive but nonlinear, and also preserve 
the positive constant functions. If $e_{\alpha}(x)=x^{\alpha}(\alpha>0)$, then $L_{n, \frac{1}{\alpha}}$ preserves $e_{\alpha}$, i.e., we get

$$
L_{n, \frac{1}{\alpha}}\left(e_{\alpha} ; x\right)=e_{\alpha}(x)=x^{\alpha} .
$$

One can also check that

$$
L_{n, \alpha}(f ; 0)=f(0) \text { and } L_{n, \alpha}(f ; 1)=f(1) \text { for any } \alpha>0 .
$$

If $0 \leq k \leq f \leq K$ on $[0,1]$ for some constants $k$ and $K$, then it is clear that

$$
k \leq L_{n, \alpha}(f) \leq K \text { on }[0,1] .
$$

Using Hölder's inequality, we also get

$$
L_{n, \frac{1}{\alpha}}(f ; x) \leq B_{n}(f, x) \leq L_{n, \alpha}(f ; x) \text { for any } \alpha \geq 1 .
$$

For a fixed $\alpha>0$, if $f^{\alpha}$ is monotonically increasing or decreasing on $[0,1]$, then so are all $L_{n, \alpha}(f)$.

\section{APPROXIMATION PROPERTIES OF NONLINEAR BERNSTEIN-TYPE OPERATORS}

Now, by $C([0,1],[0,+\infty))$ we denote the class of all non-negative and continuous functions on $[0,1]$.

Theorem 1. For any $f \in C([0,1],[0,+\infty))$ and $\alpha>0$, we have $L_{n, \alpha}(f) \rightrightarrows f$ on $[0,1]$, where the symbol $\rightrightarrows$ denotes the uniform convergence.

Proof. Let $f \in C([0,1],[0,+\infty))$ and $\alpha>0$. Assuming $0 \leq f \leq K$ on the interval $[0,1]$ define the function $g:\left[0, K^{\alpha}\right] \rightarrow[0, K]$ by $g(y)=y^{1 / \alpha}$. Then, by the uniform continuity of $g$ on $\left[0, K^{\alpha}\right]$ and the uniform convergence $B_{n}\left(f^{\alpha}\right)$ to $f^{\alpha}$ on $[0,1]$, we observe that $g \circ B_{n}\left(f^{\alpha}\right)$ is uniformly convergent to $g \circ f^{\alpha}$ on the interval $[0,1]$. This means that $L_{n, \alpha}(f) \rightrightarrows f$ on $[0,1]$.

In Figure 1, it is indicated an approximation to the function

$$
f(x)=\frac{1}{2}+\frac{1}{2} \cos \left(\frac{5 \pi x}{2}\right)
$$

by means of the operators $L_{n, 3}(f ; x)$ for $n=15,30,50,100$, respectively.

On the other hand, it follows from the general Leibniz rule

$$
(u . v)^{(k)}=\sum_{i=0}^{k}\left(\begin{array}{l}
k \\
i
\end{array}\right) u^{(k-i)} v^{(i)}
$$

that

$$
\begin{aligned}
\left(u_{1} u_{2} \ldots u_{m}\right)^{(k)}= & \sum_{i_{1}=0}^{k} \sum_{i_{2}=0}^{i_{1}} \ldots \sum_{i_{m-1}=0}^{i_{m-2}}\left(\begin{array}{c}
k \\
i_{1}
\end{array}\right)\left(\begin{array}{l}
i_{1} \\
i_{2}
\end{array}\right) \ldots\left(\begin{array}{c}
i_{m-2} \\
i_{m-1}
\end{array}\right) \\
& \times u_{1}^{\left(k-i_{1}\right)} u_{2}^{\left(i_{1}-i_{2}\right)} \ldots u_{m-1}^{\left(i_{m-2}-i_{m-1}\right)} u_{m}^{\left(i_{m-1}\right)},
\end{aligned}
$$




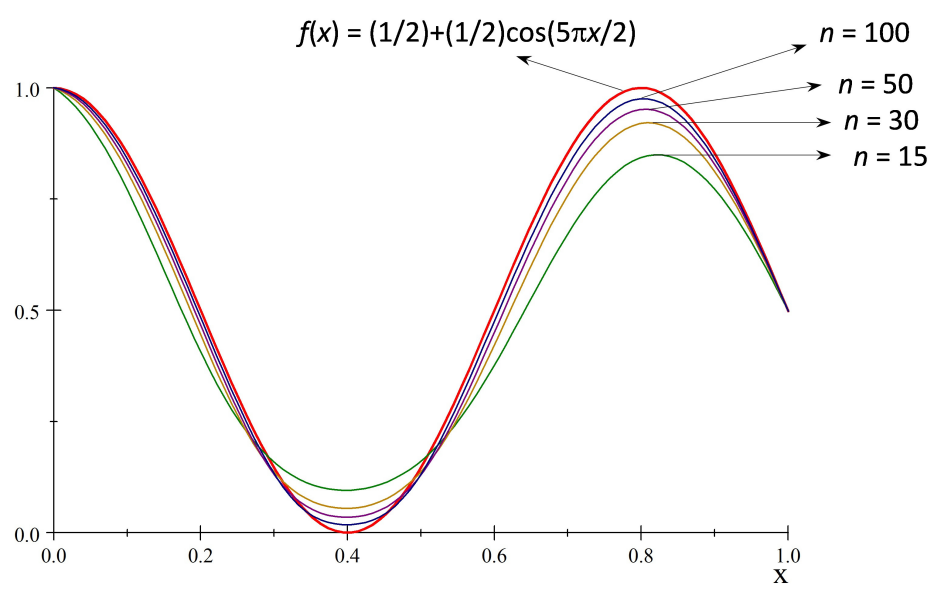

FIgURE 1. Approximation to $f(x)=\frac{1}{2}+\frac{1}{2} \cos \left(\frac{5 \pi x}{2}\right)$ by $L_{n, 3}(f ; x)$ for $n=15,30,50,100$, respectively.

where, as usual, $f^{(k)}=\frac{d^{k} f}{d x^{k}}$. Now using this, we obtain the following simultaneous approximation result for $L_{n, \alpha}(f)$.

Theorem 2. If $\alpha=1 / m, m \in \mathbb{N}$, then, we get $\left\{L_{n, \frac{1}{m}}(f)\right\}^{(k)} \rightrightarrows f^{(k)}$ on $[0,1]$ for every $f \in C^{k}([0,1],[0,+\infty))$ with $k \leq n m$.

Proof. First observe that $L_{n, \frac{1}{m}}(f ; x)$ is a polynomial with degree $\leq n m$. Then, for each $k \leq n m$, we may write that

$$
\begin{aligned}
\left\{L_{n, \frac{1}{m}}(f)\right\}^{(k)}= & \left\{\left(B_{n}\left(f^{1 / m}\right)\right)^{m}\right\}^{(k)} \\
= & \sum_{i_{1}=0}^{k} \sum_{i_{2}=0}^{i_{1}} \ldots \sum_{i_{m-1}=0}^{i_{m-2}}\left(\begin{array}{c}
k \\
i_{1}
\end{array}\right)\left(\begin{array}{l}
i_{1} \\
i_{2}
\end{array}\right) \ldots\left(\begin{array}{c}
i_{m-2} \\
i_{m-1}
\end{array}\right) \\
& \times\left\{B_{n}\left(f^{1 / m}\right)\right\}^{\left(k-i_{1}\right)}\left\{B_{n}\left(f^{1 / m}\right)\right\}^{\left(i_{1}-i_{2}\right)} \\
& \times \ldots\left\{B_{n}\left(f^{1 / m}\right)\right\}^{\left(i_{m-2}-i_{m-1}\right)}\left\{B_{n}\left(f^{1 / m}\right)\right\}^{\left(i_{m-1}\right)} .
\end{aligned}
$$

Since $\left\{B_{n}(f)\right\}^{(k)} \rightrightarrows f^{(k)}$ on $[0,1]$, we see that

$$
\left\{L_{n, \frac{1}{m}}(f)\right\}^{(k)} \rightrightarrows \sum_{i_{1}=0}^{k} \sum_{i_{2}=0}^{i_{1}} \ldots \sum_{i_{m-1}=0}^{i_{m-2}}\left(\begin{array}{l}
k \\
i_{1}
\end{array}\right)\left(\begin{array}{l}
i_{1} \\
i_{2}
\end{array}\right) \ldots\left(\begin{array}{l}
i_{m-2} \\
i_{m-1}
\end{array}\right)
$$




$$
\begin{aligned}
& \times\left\{\left(f^{1 / m}\right)\right\}^{\left(k-i_{1}\right)}\left\{\left(f^{1 / m}\right)\right\}^{\left(i_{1}-i_{2}\right)} \\
& \times \ldots\left\{\left(f^{1 / m}\right)\right\}^{\left(i_{m-2}-i_{m-1}\right)}\left\{\left(f^{1 / m}\right)\right\}^{\left(i_{m-1}\right)} \\
& =(\underbrace{f^{1 / m} f^{1 / m} \ldots f^{1 / m} f^{1 / m}}_{m \text { times }}) \\
& =f^{(k)},
\end{aligned}
$$

which completes the proof.

Here, we give the following conjecture:

Conjecture 1. For any $\alpha>0$ and $f \in C^{k}([0,1],[0,+\infty)),\left\{L_{n, \alpha}(f)\right\}^{(k)} \rightrightarrows f^{(k)}$ on $[0,1]$.

We also get the following approximation results.

Theorem 3. If $\alpha=1 / m, m \in \mathbb{N}$, then, we get, for every $n \in \mathbb{N}$ and $f \in C([0,1],[0,+\infty))$, that

$$
\left\|L_{n, \frac{1}{m}}(f)-f\right\| \leq \frac{5}{4} m K^{\frac{m-1}{m}} \omega\left(f^{1 / m}, \frac{1}{\sqrt{n}}\right),
$$

where $K:=\|f\|$ and $\omega$ denotes the classical modulus of continuity of $f$.

Proof. Using the identity

$$
u^{m}-v^{m}=(u-v)\left(u^{m-1}+u^{m-2} v+\ldots+u v^{m-2}+v^{m-1}\right),
$$

we see that

$$
\begin{aligned}
\left|L_{n, \frac{1}{m}}(f ; x)-f(x)\right|= & \left|\left(B_{n}\left(f^{1 / m} ; x\right)\right)^{m}-\left(f^{1 / m}(x)\right)^{m}\right| \\
\leq & \left|B_{n}\left(f^{1 / m} ; x\right)-f^{1 / m}(x)\right| \\
& \times \sum_{i=1}^{m}\left(B_{n}\left(f^{1 / m} ; x\right)\right)^{m-i}\left(f^{1 / m}(x)\right)^{i-1} .
\end{aligned}
$$

Since $0 \leq f(x) \leq K$ for every $x \in[0,1]$, we obtain that

$$
\left|L_{n, \frac{1}{m}}(f ; x)-f(x)\right| \leq m K^{\frac{m-1}{m}}\left|B_{n}\left(f^{1 / m} ; x\right)-f^{1 / m}(x)\right| .
$$

Now taking supremum over $x \in[0,1]$ and also using the approximation order of the classical Bernstein polynomials for the function $f^{1 / m}$, the proof is completed. 
Theorem 4. If $\alpha=m, m \in \mathbb{N}$, then, we get, for every $n \in \mathbb{N}$ and $f \in C([0,1],[0,+\infty))$ for which $\|f\| \geq k>0$, that

$$
\left\|L_{n, m}(f)-f\right\| \leq \frac{5}{4 m k^{\frac{m-1}{m}}} \omega\left(f^{m}, \frac{1}{\sqrt{n}}\right) .
$$

Proof. In (2.1) if we replace $u$ and $v$ with $u^{1 / m}$ and $v^{1 / m}$, respectively, then we have

$$
u^{1 / m}-v^{1 / m}=\frac{u-v}{u^{\frac{m-1}{m}}+u^{\frac{m-2}{m}} v^{\frac{1}{m}}+\ldots+u^{\frac{1}{m}} v^{\frac{m-2}{m}}+v^{\frac{m-1}{m}}} .
$$

Using the last equality, we see that

$$
\begin{aligned}
\left|L_{n, m}(f ; x)-f(x)\right| & =\left|\left(B_{n}\left(f^{m} ; x\right)\right)^{1 / m}-\left(f^{m}(x)\right)^{1 / m}\right| \\
& =\frac{\left|B_{n}\left(f^{m} ; x\right)-f^{m}(x)\right|}{\sum_{i=1}^{m}\left(B_{n}\left(f^{m} ; x\right)\right)^{\frac{m-i}{m}}\left(f^{m}(x)\right)^{\frac{i-1}{m}}} \\
& \leq \frac{1}{m k^{\frac{m-1}{m}}}\left|B_{n}\left(f^{m} ; x\right)-f^{m}(x)\right| \\
& \leq \frac{5}{4 m k^{\frac{m-1}{m}}} \omega\left(f^{m}, \frac{1}{\sqrt{n}}\right),
\end{aligned}
$$

whence the result.

Thus, the following open problem arises.

Open Problem. As in Theorems 3 and 4, can we get an approximation order for any $\alpha>0$ ?

The next result is useful when approximating a function $f$ for which $f^{1 / \alpha}(\alpha \geq 1)$ is convex.

Theorem 5. If $\alpha \geq 1$ and $f \in C([0,1],[0,+\infty))$ such that $f^{1 / \alpha}$ is convex on $[0,1]$, then the error estimate $\left|L_{n, \frac{1}{\alpha}}(f ; x)-f(x)\right|$ is better than $\left|B_{n}(f ; x)-f(x)\right|$.

Proof. It is well-known that if $f^{1 / \alpha}$ is convex, then $B_{n}\left(f^{1 / \alpha}\right)$ is also convex, and $B_{n}\left(f^{1 / \alpha} ; x\right) \geq f^{1 / \alpha}(x)$ for every $x \in[0,1]$ (see, for instance, [3]). Thus, we get $L_{n, \frac{1}{\alpha}}(f ; x) \geq f(x)$. Furthermore, it follows from (1.2) that

$$
f(x) \leq L_{n, \frac{1}{\alpha}}(f ; x) \leq B_{n}(f ; x) \text { for any } \alpha \geq 1,
$$

whence the result. 


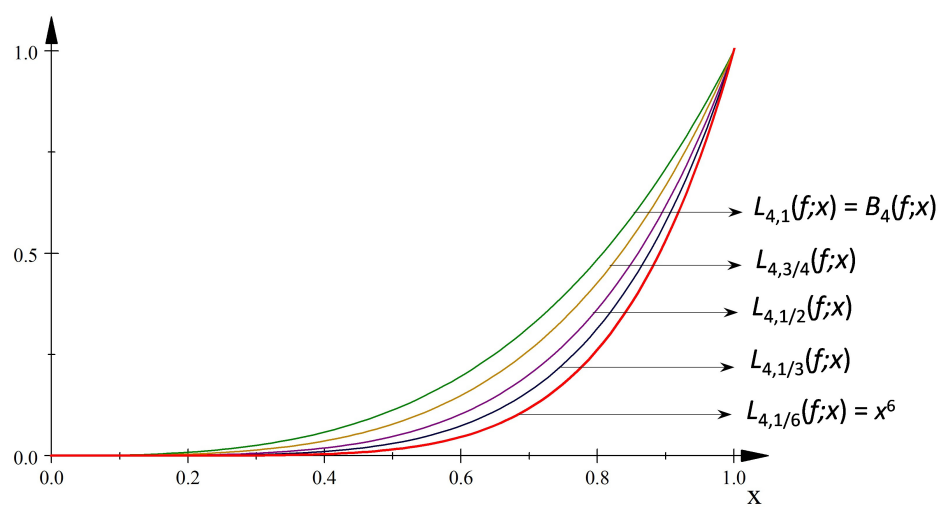

FIGURE 2. Approximation to $f(x)=x^{6}$ by $L_{4, \frac{1}{\alpha}}(f ; x)$ for $\alpha=$ $1, \frac{4}{3}, 2,3,6$, respectively.

In Figure 2, taking $n=4$ and $\alpha=1, \frac{4}{3}, 2,3,6$, respectively, we graph $L_{4, \frac{1}{\alpha}}(f)$ for the function $f(x)=x^{6}$. Then, we observe that

$$
\begin{aligned}
f(x) & =x^{6}=L_{4, \frac{1}{6}}(f ; x)<L_{4, \frac{1}{3}}(f ; x) \\
& <L_{4, \frac{1}{2}}(f ; x)<L_{4, \frac{3}{4}}(f ; x) \\
& <L_{4,1}(f ; x)=B_{4}(f ; x) .
\end{aligned}
$$

Thus, in this example, for fixed $x, n$, we see that $L_{n, \alpha}(f ; x)$ is getting close to $f(x)=x^{6}$ as $\alpha$ goes to $\frac{1}{6}$. Notice that $f^{1 / \alpha}$ is convex for $0<\alpha \leq 6$ and concave for $\alpha>6$.

Similar result also holds for the approximation to concave functions.

Theorem 6. If $\alpha \geq 1$ and $f \in C([0,1],[0,+\infty))$ such that $f^{\alpha}$ is concave on $[0,1]$, then the error estimate $\left|L_{n, \alpha}(f ; x)-f(x)\right|$ is better than $\left|B_{n}(f ; x)-f(x)\right|$.

We get from Theorem 6 and Figure 3 that, for $n=7$,

$$
\begin{aligned}
f(x) & =\sin ^{1 / 4}(\pi x)>L_{7,4}(f ; x) \\
& >L_{7,3}(f ; x)>L_{7,2}(f ; x) \\
& >L_{7,1}(f ; x)=B_{7}(f ; x) .
\end{aligned}
$$

After Theorems 5 and 6, the following problem arises:

Open Problem. Is there any other class of functions satisfying a better error estimation as in Theorems 5 and 6 ? 


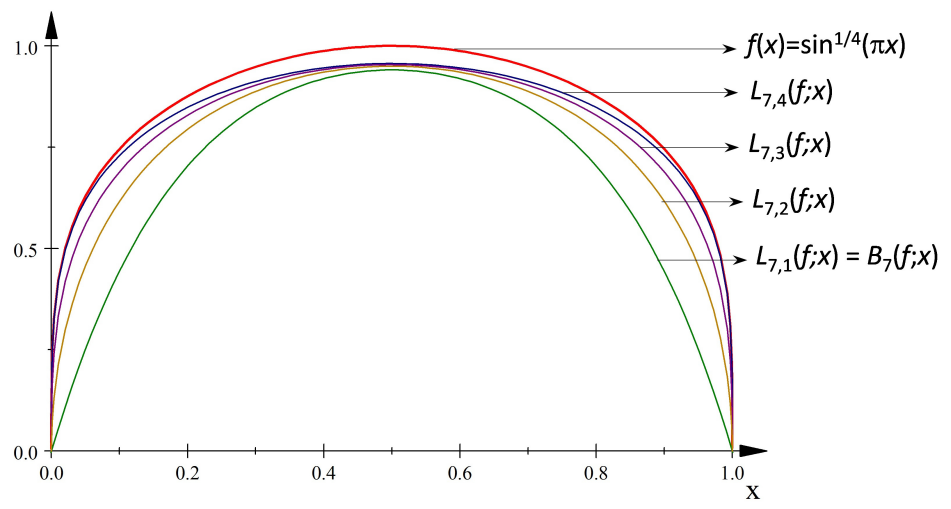

FIGURE 3. Approximation to $f(x)=\sin ^{1 / 4}(\pi x)$ by $L_{7, \alpha}(f ; x)$ for $\alpha=1,2,3,4$, respectively.

\section{EXTENSION TO THE MULTIVARIATE CASE}

Our idea can be applied to the multivariate Bernstein polynomials in the following way. We first consider the standard unit simplex in $\mathbb{R}^{m}(m \in \mathbb{N})$ :

$$
S_{m}=\left\{\left(x_{1}, \ldots, x_{m}\right): 0 \leq x_{i} \leq 1(i=1, \ldots, m) \text { and } x_{1}+\cdots+x_{m} \leq 1\right\} .
$$

Now, instead of Cartesian coordinates, we denote barycentric coordinates by a boldface symbol:

$$
\mathbf{x}=\left(x_{0}, x_{1}, \ldots, x_{m}\right) \text { with } x_{0}:=1-x_{1}-x_{2}-\cdots-x_{m} .
$$

Then, using the multi-index notations

$$
\begin{aligned}
\mathbf{x}^{\mathbf{k}} & =x_{0}^{k_{0}} x_{1}^{k_{1}} \cdots x_{m}^{k_{m}}, \\
|\mathbf{k}| & =k_{0}+k_{1}+\cdots+k_{m}, \\
\left(\begin{array}{l}
n \\
\mathbf{k}
\end{array}\right) & =\frac{n !}{k_{0} ! k_{1} ! \cdots k_{m} !},
\end{aligned}
$$

for $\mathbf{x} \in \mathbb{R}^{m+1}$ and $\mathbf{k}=\left(k_{0}, k_{1}, \ldots, k_{m}\right) \in \mathbb{N}_{0}^{m+1}$, we define the following (nonlinear) multivariate operators:

$$
L_{n, \alpha}(f ; \mathbf{x})=\left\{\sum_{|\mathbf{k}|=n}\left(\begin{array}{l}
n \\
\mathbf{k}
\end{array}\right) \mathbf{x}^{\mathbf{k}} f^{\alpha}\left(\frac{\mathbf{k}}{n}\right)\right\}^{1 / \alpha},
$$

where $f: S_{m} \rightarrow[0,+\infty)$ is a function, $n \in \mathbb{N}$ and $\alpha>0$.

In this case, as in Theorem 1, one can get the next result, immediately. 
Theorem 7. For any $f \in C\left(S_{m},[0,+\infty)\right)$ and $\alpha>0$, we have $L_{n, \alpha}(f ; \mathbf{x}) \rightrightarrows$ $f(\mathbf{x})$ on $S_{m}$.

The bivariate version of (3.1) with Cartesian coordinates may be written as follows:

$$
L_{n, \alpha}(f ; x, y)=\left\{\sum_{k=0}^{n} \sum_{j=0}^{n-k} \frac{n ! x^{j} y^{k}(1-x-y)^{n-j-k}}{j ! k !(n-j-k) !} f^{\alpha}\left(\frac{j}{n}, \frac{k}{n}\right)\right\}^{1 / \alpha}
$$

where $(x, y) \in S_{2}, n \in \mathbb{N}, \alpha>0$ and $f \in C\left(S_{2},[0,+\infty)\right)$.

\section{ACKNOWLEDGEMENT}

The author would like to thank the referee(s) for carefully reading the manuscript.

\section{REFERENCES}

[1] S. N. Bernstein, "Démonstration du théorème de weierstrass, fondeé sur le calcul des probabilités," Commun. Soc. Math. Kharkow, vol. 13, pp. 1-2, 1912.

[2] G. G. Lorentz, Bernstein Polynomials, 2nd ed. New York: Chelsea Publishing Co, 1986.

[3] G. M. Phillips, Interpolation and Approximation by Polynomials, ser. CMS Books in Mathematics. New York: Springer-Verlag, 2003, vol. 14.

\section{Author's address}

\section{Oktay Duman}

TOBB Economics and Technology University, Department of Mathematics, Söğütözü TR-06530, Ankara, Turkey

E-mail address: oduman@etu.edu.tr; okitayduman@gmail.com 